\title{
AN APPLICATION OF EIGENVALUE ESTIMATE TO ALGEBRAIC CURVES DEFINED BY CONGRUENCE SUBGROUPS
}

\author{
SHING-TUNG YAU
}

\section{$\S 1$. Arithmetic curves}

Let $X$ be an algebraic curve defined by a congruence subgroup. Then it is clear that $X$ enjoys many special properties as an algebraic curve. How to characterize it algebraic geometrically? Ten years ago, based on my work with Peter $\mathrm{Li}$ [7], the author made the simple observation that the degree of this curve over the projective line must be large, i.e., at least a (universal) constant multiple of the genus. In particular, there is an explicit bound of the genus for such a curve which is hyperelliptic. It is also interesting to understand a similar estimate for the degree of this curve over an elliptic curve. Since there is interest in such estimates, we decided to publish this note.

Let $\Sigma$ be any algebraic curve. In [7] Peter Li and I defined an invariant attached to its complex structure in the following way. Let

$$
A_{C}(\Sigma)=\inf _{F} \sup _{g} \operatorname{Area}(g(F(\Sigma)))
$$

where $F$ is any nontrivial branched conformal map of $\Sigma$ into a unit sphere $S^{n}$ and $g$ is any element in the conformal group of $S^{n}$.

$\mathrm{Li}$ and the author called $A_{C}(\Sigma)$ to be the conformal area of $\Sigma$. It can be computed for cases when $\Sigma$ can be conformal immersed into $S^{n}$. In particular, it is equal to $4 \pi$ when $\Sigma=S^{2}=\mathbb{C} P^{1}$. We proved that for any conformal metric defined on $X$, the first eigenvalue $\lambda_{1}$ of the Laplacian is dominated by

$$
\lambda_{1} \leq \frac{2 d A_{C}(\Sigma)}{\operatorname{Area}(X)}
$$

Received December 22, 1995.

Supported in part by NSF Grant DMS-9504834. 
where $d$ is the minimal degree of a holomorphic map onto $\Sigma$. (See also [9] where Paul Yang and the author studied the case where $\Sigma$ is mapped to $S^{2}$.)

In such an inequality, if we take the metric to be the Poincaré metric, Area $(X)=4 \pi(g-1)$ and by Selberg's theorem, we have the following.

Theorem 1. Let $X$ be any curve defined by the congruence subgroup. Then for any holomorphic map from $X$ to an algebraic curve $\Sigma$ with degree $d$, we have the following inequality

$$
d A_{C}(\Sigma) \geq \frac{3 \pi}{8}\left(g-1+\frac{n}{2}\right)
$$

where $g$ is the genus of $X$ and $n$ is the number of ends of $X$.

Corollary. The degree of any $X$ mapping to $\mathbb{C} P^{1}$ must be greater than $\frac{3}{32}\left(g-1+\frac{n}{2}\right)$.

Note that if $\lambda_{1} \geq \frac{1}{4}$, the inequality will be improved to $\frac{1}{8}\left(g-1+\frac{n}{2}\right)$. Hence the genus of any $X$ which is hyperelliptic must be less than 17 .

If we consider the Laplacian acting on a (flat) complex line bundle $L$ over $X$, we can also find the upper bound for the first eigenvalue. Let us treat the simplest case where the line bundle is defined by a representation of the fundamental group of $X$ to $Z_{p}$. Let $\omega$ be the $p$-th root of unity. Then $\omega$ acts on $S^{2 n-1} \subset \mathbb{C}^{n}$ diagonally. There is an obvious flat $Z_{p}$ line $\widetilde{L}$ bundle over $S^{2 n-1} / Z_{p}$. Let $F$ be any branched conformal inversion of $X$ into $S^{2 n-1} / Z_{p}$ so that the pullback of $\widetilde{L}$ is equal to $L$. The complex coordinates of $S^{2 n-1}$ defines a section of $\widetilde{L}$. Hence we can apply the argument of Li-Yau to conclude that the first eigenvalue of $L$ over $X$ is bounded by

$$
\lambda_{1}(L) \leq 2(\text { Area } X)^{-1} \inf _{F}(\text { Area } F) .
$$

For $n=1$, we should understand this inequality as follows: Take any one form $\omega$ with integral period and some period equal to one. It defines a map from $X$ into the circle. Then

$$
\lambda_{1}(L) \leq \frac{4 \pi^{2}}{p^{2}} \frac{\inf _{\omega}\|\omega\|^{2}}{\text { Area } X} .
$$

When $L$ is trivial, the first eigenvalue considered here is zero. (The previous first eigenvalue should be considered as first non-trivial eigenvalue.) 
The estimate on the first eigenvalue shows that when

$$
d<\frac{1}{8}\left(g-1+\frac{n}{2}\right)
$$

there is a discrete eigenvalue below $\frac{1}{4}$. It is interesting to know how many eigenvalues are less than $\frac{1}{4}$. In fact, answering a question of the author, $\mathrm{N}$. Korevare [4] proved that

$$
\lambda_{i} \leq C \operatorname{di}(A(\Sigma))^{-1}
$$

where $C$ is a universal constant. (The best constant is still not known.)

Hence if $m$ is the smallest integer less than $\frac{\pi\left(g-1+\frac{n}{2}\right)}{c d}$, there are $m$ $L^{2}$-eigenfunctions with eigenvalue less than $\frac{1}{4}$.

Let us now assume that we are dealing with a compact curve $X$. It is a theorem due to Onofri [5], and Osgood-Philips-Sarnak [6] that among metrics with the same area, the determinant of the Laplacian is maximum when the curvature of the metric is constant. If there is a holomorphic map from $X$ onto $S^{2}$ with degree $d$, we can pull back the metric from $S^{2}$ to $X$. The determinant of the Laplacian of the pulled back metric is the $d$-th power of the determinant of the Laplacian of $S^{2}$.

Hence if we normalize the curvature of $S^{2}$ so that $4 \pi d^{-1}(g-1)$ is equal to the area of $S^{2}$, then the determinant of the Laplacian of $X$ is dominant by the $d$-th power of the Laplacian of $S^{2}$. It is not clear how to obtain a good lower bound for the determinant of the Laplacian for $X$ defined by arithmetic means.

If $X$ has dimension greater than one, there is an upper estimate of the first (non-trivial) eigenvalue also. In fact, if $X$ is an $n$-dimensional Kähler manifold with Kähler class $\omega$ and if $f$ is any holomorphic map from $X$ to $\mathbb{C} P^{1}$, then $\lambda_{1}(X)$ is dominated by $2 \int_{M}\left(f^{*} \Omega \wedge \omega^{n-1}\right) \operatorname{Vol}^{-1}(M)$ where $\Omega$ is the standard Kähler form of $\mathbb{C} P^{1}$. For higher dimensional manifolds, it is natural to consider holomorphic maps from $X$ into $\mathbb{C} P^{N}$. In this case Bourguignon, Li and myself [1] prove that the first eigenvalue is less than

$$
m \frac{N+1}{N}\left(\int f^{*}(\Omega) \wedge \omega^{m-1}\right) \mathrm{Vol}^{-1}(M)
$$


Since there should be a universal lower estimate for algebraic manifolds defined by arithmetic groups acting on Hermitian symmetric space of noncompact type, there should be lower estimates of the degree $\int f^{*}(\Omega) \wedge \omega^{m-1}$ for these manifolds in terms of $\operatorname{Vol}(M)$. Note that $\omega$ can be taken to be $-c_{1}(M)$ and $\operatorname{Vol}(M)$ is simply $(-1)^{m} c_{1}^{m}(M)$. Hence if $\lambda_{1}$ has a good lower bound, the line bundle that embeds $X$ into a complex projective space cannot have low degree relative to the canonical line bundle.

It is an interesting question to generalize the estimate of N. Korevare to higher dimensional Kähler manifolds. Perhaps there is also some estimate for holomorphic torsion similar to the determinant of the Laplacian of an algebraic curve.

For the original problem of characterizing the curve defined by arithmetic group, one may mention that there is the famous work of Belli on characterizing algebraic curve defined over $\bar{Q}$. Perhaps the structure of the Hecke algebra can be used to give further conditions.

\section{$\S 2$. Theta function}

Let $T^{g}$ be a real torus defined by a lattice $L$ with $g>2$. Let

$$
\theta_{L}(t)=\sum_{\ell \in L} \exp \left(\frac{-\|\ell\|^{2}}{4 t}\right) .
$$

Based on eigenvalue estimate mentioned in the last section, we shall derive some special property of $\theta(t)$ when $T^{g}$ contains a minimal surface $\Sigma$. The function

$$
\widetilde{h}(t, x, y)=\frac{1}{4 \pi t} \sum_{\ell \in L} \exp \left(-\frac{\|x-y+\ell\|^{2}}{4 t}\right)
$$

defines a function on the surface $\Sigma$. We shall use $\widetilde{h}$ to compare the heat kernel of $\Sigma$ with respect to the induced metric on $\Sigma$. Since $\Sigma$ is a minimal surface, $\Delta_{\Sigma} X=0$ when $X$ is considered as a function defined on $\Sigma$. It follows that $\frac{\partial \hbar}{\partial t}-\Delta \widetilde{h} \geq 0$. The standard argument as was shown in [2][3] then shows that $\widetilde{h}(t, x, y) \geq h(t, x, y)$.

In particular, setting $x=y$, we obtain

$$
\frac{1}{4 \pi t} \sum_{\ell \in L} \exp \left(-\frac{\|\ell\|^{2}}{4 t}\right) \geq \sum_{i=0}^{\infty} e^{-\lambda_{i} t} \varphi_{i}^{2}(x)
$$


where $\varphi_{i}$ are the $i$-th eigenfunctions of the Laplacian $\Delta_{\Sigma}$ such that $\int_{\Sigma} \varphi_{i}^{2}=1$

Since $\lambda_{i} \geq C \operatorname{di}(A(\Sigma))^{-1}$, we deduce that

$$
\theta_{L}(t) \geq \frac{4 \pi t}{A(\Sigma)}\left[1-\exp \left(\frac{-C d t}{A(\Sigma)}\right)\right]^{-1}
$$

where $A(\Sigma)$ is the area of $\Sigma$.

Theorem 2.1. If a real torus $T^{g}$ contains a minimal surface $\Sigma$ whose degree is $d$, then its theta function $\theta$ of $T^{g}$ satisfies the inequality (2.4).

Note that algebraic curves are minimal surfaces. Hence we can apply the above theorem to Jacobian of a curve and $A(\Sigma)$ can be computed in terms of the genus.

By the Poisson formula, $\theta_{L^{*}}\left(\frac{1}{4 t}\right)=(4 \pi t)^{-g / 2} \theta_{L}(t) \operatorname{Vol}\left(T^{g}\right)$, where $L^{*}$ is the lattice dual to $L$. It follows from (2.4) that

$$
\sum_{\ell \in L^{*}} \exp \left(-t\|\ell\|^{2}\right) \geq \frac{(4 \pi t)^{\frac{-g}{2}+1} \operatorname{Vol}\left(T^{g}\right)}{A(\Sigma)}\left[1-\exp \left(\frac{-C d t}{A(\Sigma)}\right)\right]^{-1} .
$$

Formula (2.5) shows that $L^{*}$ contains nontrivial elements of small length.

Coming back to $(2.4)$, notice that $(4 \pi t)^{-g / 2} \theta_{L}(t)$ is the heat kernel of $T^{g}$ along the diagonal. Hence according to the upper estimate of the heat kernel provided in [8], we have

$$
(4 \pi t)^{-g / 2} \theta_{L}(t) \leq C^{\prime} \operatorname{Vol}(B(\sqrt{t}))^{-1}
$$

where $C^{\prime}$ is a constant depending only on $g$ and $B(\sqrt{t})$ is any geodesic ball with radius $\sqrt{t}$ in $T^{g}$.

Combining (2.4) and (2.6) we obtain

$$
C^{\prime} \frac{(4 \pi t)^{g / 2}}{B(\sqrt{t})} \geq \frac{4 \pi t}{A(\Sigma)}\left[1-\exp \left(\frac{-C d t}{A(\Sigma)}\right)\right]^{-1}
$$

Let $\sqrt{t}=D$ to be the diameter of $T^{g}$, we obtain the following inequality

$$
C^{\prime}(4 \pi D)^{g} \operatorname{Vol}\left(T^{g}\right)^{-1} \geq \frac{4 \pi D^{2}}{A(\Sigma)}\left[1-\exp \left(\frac{-C d D^{2}}{A(\Sigma)}\right)\right]^{-1}
$$


Inequality (2.8) shows that if $D^{2}(A(\Sigma))^{-1}$ is large, the lattice defining $T^{g}$ cannot be close to the standard lattice.

The inequality (2.3) easily implies the following statement for the $L^{\infty}$ norm of eigenfunction

$$
\begin{aligned}
\frac{1}{m} \sum_{i=1}^{m} \varphi_{i}^{2}(x) & \leq \frac{\lambda_{m} e}{4 \pi m} \theta\left(\frac{1}{\lambda_{m}}\right) \\
& \leq \frac{C d e}{4 \pi A(\Sigma)} \theta\left(\frac{1}{\lambda_{m}}\right) .
\end{aligned}
$$

The inequality $\widetilde{h}(t, x, y) \geq h(t, x, y)$ can also be improved to the following sharper inequality

$$
\iint_{\Sigma} \int \widetilde{h}\left(\alpha_{1} t, x, z_{1}\right) \widetilde{h}\left(\alpha_{2} t, z_{1}, z_{2}\right) \cdots \widetilde{h}\left(\alpha_{k} t, z_{k-1}, y\right) d z_{1} \cdots d z_{k-1} \geq h(t, x, y)
$$

where $\alpha_{i}$ are positive constants with $\alpha_{1}+\cdots+\alpha_{k}=1$.

\section{References}

1. J. P. Bourguignon, P. Li, and S. T. Yau, Upper bound for the first eigenvalue of algebraic submanifolds, Publ. C.N.R.S., U.R.A. 169, no. 1057 (1993).

2. J. Cheeger and S. T. Yau, A lower bound for the heat kernel, Comm. on Pure Appl. Math. 34; no. 4 (1981), 465-480.

3. S. Y. Cheng, P. Li, and S. T. Yau, Heat euqations on minimal submanifolds and their applications, Amer. J. Math. 106 (1984), 1033-1065.

4. N. Korevaar, Upper bounds for eigenvalues of conformal metrics, J. Diff. Geom. 37 (1993), 73-93.

5. E. Onofri, On the positivity of the effective action in a theory of random surfaces, Comm. Math. Phys. 86 (1982), 321-326.

6. B. Osgood, R. Phillips, and P. Sarnak, Extremals of determinants of Laplacians, J. Funct. Anal. 80 (1988), 148-211.

7. P. Li and S.-T. Yau, A new conformal invariant and its applications to the Willmore conjecture and the first eigenvalue of compact surfaces, Invent. Math. 69 (1982), 269-291.

8. 153-201.

9. P. C. Yang and S.-T. Yau, Eigenvalues of the Laplacian of compact Riemann surfaces and minimal submanifolds, Ann. Scuola Norm. Sup. Pisa 7 (1980), 55-63.

Department of Mathematics, Harvard Univeritiy, Cambridge, M A 02138 , AND Chinese Univerity OF Hong Kong

E-mail address: yau@math.harvard.edu 